Sādhanā Vol. 40, Part 7, October 2015, pp. 2111-2128. (C) Indian Academy of Sciences

\title{
Weighted-noise threshold based channel estimation for OFDM systems
}

\author{
PALLAVIRAM SURE ${ }^{1, *}$ and CHANDRA MOHAN BHUMA ${ }^{2}$ \\ ${ }^{1}$ Acharya Nagarjuna University, Guntur, Andhra Pradesh 522510, India \\ ${ }^{2}$ Bapatla Engineering College, Guntur, Andhra Pradesh 522101, India \\ e-mail: pallaviram.sure@yahoo.com; chandrabhuma@gmail.com
}

MS received 29 January 2015; revised 28 May 2015; accepted 23 June 2015

\begin{abstract}
Orthogonal frequency division multiplexing (OFDM) technology is the key to evolving telecommunication standards including 3GPP-LTE Advanced and WiMAX. Reliability of any OFDM system increases with improved mean square error performance (MSE) of its channel estimator (CE). Particularly, a least squares (LS) based CE incorporating a time-domain denoising threshold, enables better MSE performance, while avoiding the need for a-priori knowledge of channel statistics (KCS). Existing optimal time-domain thresholds exhibit suboptimal behavior for completely unavailable KCS environments. This is because they involve consistent estimation of one or more KCS parameters, and corresponding estimation errors introduce severe degradation in MSE performance of the CE. To overcome the MSE degradation, this paper proposes a weighted-noise threshold, by introducing a modified hypothesis-testing-problem (HTP) interpretation. Derivation of resulting analytical MSE expression is also provided. Results of OFDM system simulations carried out in rayleigh faded ITU-TU6 and WiMAX-SUI4 channel environments with U-shaped power spectral densities, are presented. The performance results show that, compared to many of the existing thresholds, the proposed threshold renders better MSE performance to the $\mathrm{CE}$ and higher reliability to the OFDM system in terms of better bit error rate $(\mathrm{BER})$ performance.
\end{abstract}

Keywords. OFDM pilot-based channel estimation; weighted-noise threshold; MSE performance.

\section{Introduction}

Orthogonal frequency division multiplexing (OFDM) technology offers numerous advantages like very high data rates, elimination of inter symbol interference (ISI) and mitigation of inter

*For correspondence 
carrier interference (ICI). Due to the orthogonality of sub-carriers, OFDM system realization involves less complexity. Hence OFDM technology is readily being incorporated in present and emerging communication systems like LTE, WLAN, WiFi and WiMAX. System reliability depends on how effective is the estimated fading channel. For this reason, normally data-aided OFDM channel estimation, either in time or in frequency domain is adopted. In time domain algorithms, preamble at the start of each OFDM frame and postfix after every OFDM symbol, help in channel estimation (Dai et al 2013). In frequency domain algorithms, cyclic prefix at the begin of every OFDM symbol and pilot symbols (known data symbols) arranged in a pre-determined fashion over the OFDM grid, help in channel estimation (Hsieh \& Wei 1998).

Frequency domain algorithms are either least-squares (LS) based or minimum mean square error (MMSE) based (Coleri et al 2002). Compared to LS based channel estimator(CE), MMSE based CE renders better MSE performance to the CE. The latter requires a-priori Knowledge of channel statistics (KCS) in the form of channel second order statistics (Coleri et al 2002) while the former does not require any a-priori KCS. Here, channel environments with unknown KCS are considered, and therefore LS based CE is incorporated. To further enhance their MSE performance, denoising thresholds are employed on the LS estimated channel response.

So far, different time-domain thresholds have been proposed in the literature based on different optimizing strategies. A threshold in time-domain distinguishes significant channel impulse response (CIR) taps from the noisy taps. Some of them are discussed as follows. In van de Beek et al (1995), the LS estimated CIR is truncated assuming known channel length, without employing a threshold. Compared with no truncation case, truncation improved the MSE performance of the CE. In Minn \& Bhargava (2000), by assuming known channel length, the significant CIR taps are selected based on a threshold, which is a fraction of known maximum channel tap's energy. This method is shown to be better than that of van de Beek et al (1995), but requires KCS in the form of channel length and maximum channel tap energy. A threshold of twice the estimated noise variance is proposed in Kang et al (2007), assuming known channel length. Its MSE performance is superior to that in van de Beek et al (1995). In Oliver et al (2008), an optimum threshold is derived by maximizing the tap-detection probability, which showed better performance than the threshold of Minn \& Bhargava (2000). However, this requires complete power profile of channel, noise variance and also channel length parameters.

Another optimal threshold is derived in Rosati et al (2012) by minimizing MSE, which renders better MSE performance compared to the thresholds derived in Kang et al (2007) and Oliver et al (2008). This threshold requires noise variance, total number of non-zero channel-taps and number of pilots (which is fixed assuming known channel length). Wavelet based threshold is proposed in Lee et al (2009), which is shown to be better than that of Kang et al (2007). A universal-threshold is proposed in Xie et al (2013), which showed better MSE performance than that of Kang et al (2007). Table 1 summarizes some of the existing threshold expressions. In this table, $L$ stands for channel length, $N_{t}$ represents total non-zero channel-taps (i.e. significant taps), $\sigma_{v}^{2}$ denotes noise variance and $N_{p}$ is the number of pilot subcarriers. The Table shows that, to incorporate a given threshold in completely unavailable KCS conditions, one or more KCS parameters need to be estimated. This estimation readily causes MSE performance of CE to degrade from the actual MSE performance (obtained when KCS is completely available). However, in the existing literature for OFDM systems, MSE performance degradation due to estimation of KCS parameters, namely $\sigma_{v}^{2}, L, N_{t}$, has not been studied. In Kashyap \& Mehta (2014), in the context of driving power control in underlay cognitive radio, impact of various channel estimation errors has been studied. Motivated by this type of study, the following are the contributions of the present paper. 
Table 1. Some existing thresholds and KCS requirements.

\begin{tabular}{lcccc}
\hline Paper & Threshold & \multicolumn{3}{c}{ KCS parameters } \\
\cline { 3 - 4 } Kang et al (2007) & $2 \sigma_{v}^{2}$ & Yes & No & Yes \\
Oliver et al (2008) & $\sigma_{v}^{2} \ln \left(2+\frac{1}{N_{t} \sigma_{v}^{2}}\right)$ & No & Yes & Yes \\
Rosati et al (2012) & $\frac{\ln \left(\frac{\left(N_{p}-N_{t}\right)}{N_{t}^{2} \sigma_{v}^{2}}\right)}{\left(\frac{1}{\sigma_{v}^{2}}-N_{t}\right)}, N_{p}>L$ & Yes & Yes & Yes \\
Xie et al (2013) & $2 \sigma_{v}^{2} \ln (L)$ & & & No \\
\hline
\end{tabular}

MSE performance degradation of some of the existing thresholds due to KCS parameter estimation is studied. To mitigate the MSE degradation, we propose a weighted-noise threshold. To derive this threshold, we propose a modified hypothesis testing-problem (HTP) interpretation of the LS estimated CIR. Unlike the existing thresholds, the proposed threshold incorporates a flexible noise-weight parameter $q$, in addition to the KCS parameters, $\sigma_{v}^{2}, L, N_{t}$. For a given channel, proper selection of $q$ balances the error due to KCS parameter estimation and thus, helps the $\mathrm{CE}$, to always maintain actual MSE performance (that obtained when KCS is completely available). An empirical formula for $q$ is devised, which is justified by the simulation results. Also, analytical MSE expression of the CE, incorporating the proposed threshold, is derived. Whether the KCS parameters are completely known or unknown, with the help of numerical results and OFDM system simulations, the proposed threshold is verified to render better MSE performance than many other existing thresholds.

The rest of the paper is organized as follows. In section 2, the adopted OFDM system model and channel estimation procedure are introduced. In section 3, a HTP interpretation is framed for the LS estimated CIR corresponding to some existing optimal thresholds. The proposed modified HTP interpretation is framed in section 4 and the derivation of the proposed threshold is presented. Analytical MSE expression with the proposed threshold is also derived in this section. Numerical results and OFDM system simulation results are presented in section 5 . The paper is finally concluded in section 6 .

\section{Adopted system model: channel estimation}

We consider an OFDM system comprising of $N$ sub-carriers (Coleri et al 2002). The transmitted $i^{\text {th }}$ OFDM-symbol is represented as $X_{i, k}, 0 \leq k \leq N-1$, where $k$ is the sub-carrier index in frequency domain. Pilot symbols are sent on a few out of $N$ sub-carriers, in any given OFDM symbol, in a periodic pattern (comb-type Coleri et al 2002). Indicating the pilot positions as $P(k)$, the pilot transmissions correspond to $X_{i, k}, k \in P(k)$ and data transmissions correspond to $X_{i, k}, k \notin P(k)$. Each of the resultant OFDM symbol is converted to time domain using inverse DFT (IDFT), and a cyclic prefix is added at the beginning of each OFDM symbol. The resultant symbols are transmitted over the wireless fading channel.

At the receiver, the cyclic prefix is first removed and a DFT operation is performed to get back the frequency domain OFDM symbols, which can be expressed as

$$
Y_{i, k}=H_{i, k} X_{i, k}+N_{i, k}, 0 \leq k \leq N-1 \text {, }
$$


where $H_{i, k}$ represents actual channel frequency response (CFR) and $N_{i, k}$ represents AWGN. The system model assumes perfect synchronization between the transmitter and receiver (Morelli \& Mengali 2001). In practice synchronization problems are resolved using the techniques described in Sheng (2014), and Larsson et al (2001). System model assumes that the channel is time-invariant within one OFDM symbol duration (Coleri et al 2002; Rosati et al 2012).

The LS based CE uses received OFDM symbol $Y_{i, k}$ and the transmitted OFDM symbol $X_{i, k}$, at the pilot positions $k \in P(k)$ to obtain the LS estimated CFR at pilot positions, shown as

$$
\hat{H}_{i, k}^{L S, p i l}=\frac{Y_{i, k}}{X_{i, k}}, k \in P(k) .
$$

The resultant CFR at pilot positions is then low-pass interpolated (Carlos Augusto et al 2007) to obtain the LS estimated CFR, $\hat{H}_{i, k}^{L S}$, for all $k \in[0, N-1]$. Then, substituting for $Y_{i, k}$ using (1), the LS estimated CFR can be expressed as

$$
\hat{H}_{i, k}^{L S}=H_{i, k}+V_{i, k}, k \in[0, N-1],
$$

where $V_{i, k}=\frac{N_{i, k}}{X_{i, k}}$. Applying IDFT on the LS estimated CFR (3), the LS estimated CIR, $\hat{h}_{i, n}^{L S}$ can be obtained as

$$
\hat{h}_{i, n}^{L S}=h_{i, n}+v_{i, n}, 0 \leq n \leq N-1,
$$

In (4), $n$ is sub-carrier index in time domain. Assuming that the number of pilot sub-carriers $N_{p}$ is larger than channel length $L$ (Rosati et al 2012), the LS estimated CIR after $n=L-1$ is forced to zero, resulting in truncated CIR given by

$$
\hat{h}_{i, n}=\left\{\begin{array}{cc}
\hat{h}_{i, n}^{L S} & 0 \leq n \leq L-1 \\
0 & L \leq n \leq N-1 .
\end{array}\right.
$$

Generally, in a wide sense stationary uncorrelated scattering (WSS-US) environment, $h_{i, n}$ is a zero-mean complex-Gaussian random variable $h_{i, n} \sim N_{c}\left(0, \gamma_{n}^{2}\right)$, where variance $\gamma_{n}^{2}$ is the average energy of $n^{\text {th }}$ channel-tap and $v_{i, n} \sim N_{c}\left(0, \sigma_{v}^{2}\right)$ (Rosati et al 2012). The final CIR obtained by applying threshold $\vartheta$ is given by

$$
\hat{h}_{i, n}^{t}=\left\{\begin{array}{cc}
\hat{h}_{i, n}, & \left|\hat{h}_{i, n}\right|^{2}>\vartheta \\
0, & \text { otherwise }
\end{array} .\right.
$$

The above-mentioned channel estimation procedure can be summarized into five steps as follows:

1. Obtain the LS estimated CFR at pilot positions using (2).

2. Interpolate (2) to obtain $\hat{H}_{i, k}^{L S}$ in (3).

3. Perform IDFT on $\hat{H}_{i, k}^{L S}$ to LS estimated CIR, $\hat{h}_{i, n}^{L S}$.

4. Truncate $\hat{h}_{i, n}^{L S}$ after $n=L-1$ as in (5).

5. Apply threshold $\vartheta$ on the truncated CIR as in (6).

In the existing literature, the LS estimated and truncated CIR $\hat{h}_{i, n}$ is explored in various ways to obtain different thresholding strategies. Two such existing optimal thresholds are discussed next to obtain some valuable interpretations. 


\section{Existing HTP and corresponding threshold}

In this section, the optimal thresholds derived in Oliver et al (2008) and Rosati et al (2012), whose expressions are shown in table 1 , are treated. Both these thresholds can be interpreted using a classical HTP interpretation of $\hat{h}_{i, n}$, given by

$$
\left\{\begin{array}{cl}
\hat{h}_{i, n}= & \\
v_{i, n} \sim N_{c}\left(0, \sigma_{v}^{2}\right) & \left|h_{i, n}\right|^{2}=0: H_{0} \\
\left(h_{i, n}+v_{i, n}\right) \sim N_{c}\left(0, \gamma_{n}^{2}+\sigma_{v}^{2}\right) & \left|h_{i, n}\right|^{2} \neq 0: H_{1} .
\end{array}\right.
$$

The HTP interpretation is understood as follows. On a given $i^{\text {th }}$ OFDM symbol, $\hat{h}_{i, n}$, for each value of $n$, where $0 \leq n \leq L-1$, may be a significant CIR tap or a zero tap. If for a given $n$, $\left|h_{i, n}\right|^{2}=0$, which implies $\hat{h}_{i, n}$ is a zero tap, then $\hat{h}_{i, n}$ belongs to hypothesis $H_{0}$, else it belongs to hypothesis $H_{1}$. To find out if every $n^{\text {th }}$ channel tap for all $0 \leq n \leq L-1$ is significant tap or a zero tap, a threshold has to be developed.

The HTP in (8) can be further extended to the HTP in (8), so as to simplify the statistical computations. The simplification occurs due to the fact: if random variable $x$ is complex Gaussian, $|x|^{2}$ is exponential and the integrations become easier in this case rather than in the case of Gaussian. In (8), E ( $\beta$ ) represents exponentially distributed random variable with parameter $\beta$, having probability density function (pdf) $f(x)=\beta e^{-\beta x}$.

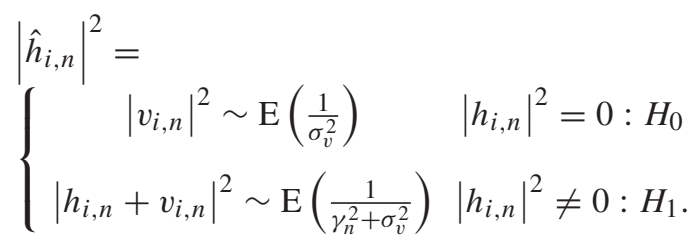

The HTP interpretation becomes useful to analyze the tap-detection problem. The HTP of (8) results in four possible courses of action, namely

$$
\begin{array}{cccc}
\text { Event } & \text { Actual } & \text { Detected } & \text { Probability } \\
I & \left|h_{i, n}\right|^{2} \neq 0\left|\hat{h}_{i, n}\right|^{2}>\vartheta & P_{D} \\
\text { II } & \left|h_{i, n}\right|^{2} \neq 0\left|\hat{h}_{i, n}\right|^{2}<\vartheta & 1-P_{D} \\
\text { III } & \left|h_{i, n}\right|^{2}=0\left|\hat{h}_{i, n}\right|^{2}>\vartheta & P_{F A} \\
\text { IV } & \left|h_{i, n}\right|^{2}=0\left|\hat{h}_{i, n}\right|^{2}<\vartheta & 1-P_{F A} .
\end{array}
$$

In (9), $P_{D}$ indicates correct-decision probability and $P_{F A}$ indicates false-alarm probability. Events $I, I V$ lead to correct-decision, while II, III are erroneous. In Oliver et al (2008), the threshold is derived by maximizing the probability, $\operatorname{Pr}\left(\left|v_{i, n}\right|^{2} \leq \vartheta<\left|h_{i, n}+v_{i, n}\right|^{2}\right)$, which is simply the product of $P_{D}\left(1-P_{F A}\right)$. 
Indicating $C$ as the set of channel-taps with $\left|h_{i, n}\right|^{2} \neq 0$, consider the corresponding MSE expression (Rosati et al 2012) of the CE written as

$$
\begin{aligned}
& M S E=\sum_{n=0}^{N-1} E\left\{\left|h_{i, n}-\hat{h}_{i, n}\right|^{2}\right\}=\sum_{n=0}^{N-1} E\left\{\left|e_{i, n}\right|^{2}\right\} \\
& =\sum_{n \in C}\left(P_{D} E\left\{\left|e_{i, n}\right|^{2} / I\right\}+\left(1-P_{D}\right) E\left\{\left|e_{i, n}\right|^{2} / I I\right\}\right) \\
& \quad+\sum_{n \notin C} P_{F A} E\left\{\left|e_{i, n}\right|^{2} / I I I\right\} .
\end{aligned}
$$

In (10), $e_{i, n}$ is the difference between actual and estimated CIR, which represents the error in the estimation of CIR. The probabilities $P_{D}$ and $P_{F A}$ of (10) are obtained from Rosati et al (2012) as

$$
\begin{aligned}
& P_{D}=\operatorname{Pr}\left\{\left|\hat{h}_{i, n}\right|^{2}>\vartheta /\left|h_{i, n}\right|^{2} \neq 0\right\}=\left(\mathrm{e}^{-\frac{\vartheta}{\gamma_{n}^{2}+\sigma_{v}^{2}}}\right) \\
& P_{F A}=\operatorname{Pr}\left\{\left|\hat{h}_{i, n}\right|^{2}>\vartheta /\left|h_{i, n}\right|^{2}=0\right\}=\left(e^{-\frac{\vartheta}{\sigma_{v}^{2}}}\right) .
\end{aligned}
$$

The first two conditional expectations are given as

$$
E\left\{\left|e_{i, n}\right|^{2} / I\right\}=\sigma_{v}^{2}
$$

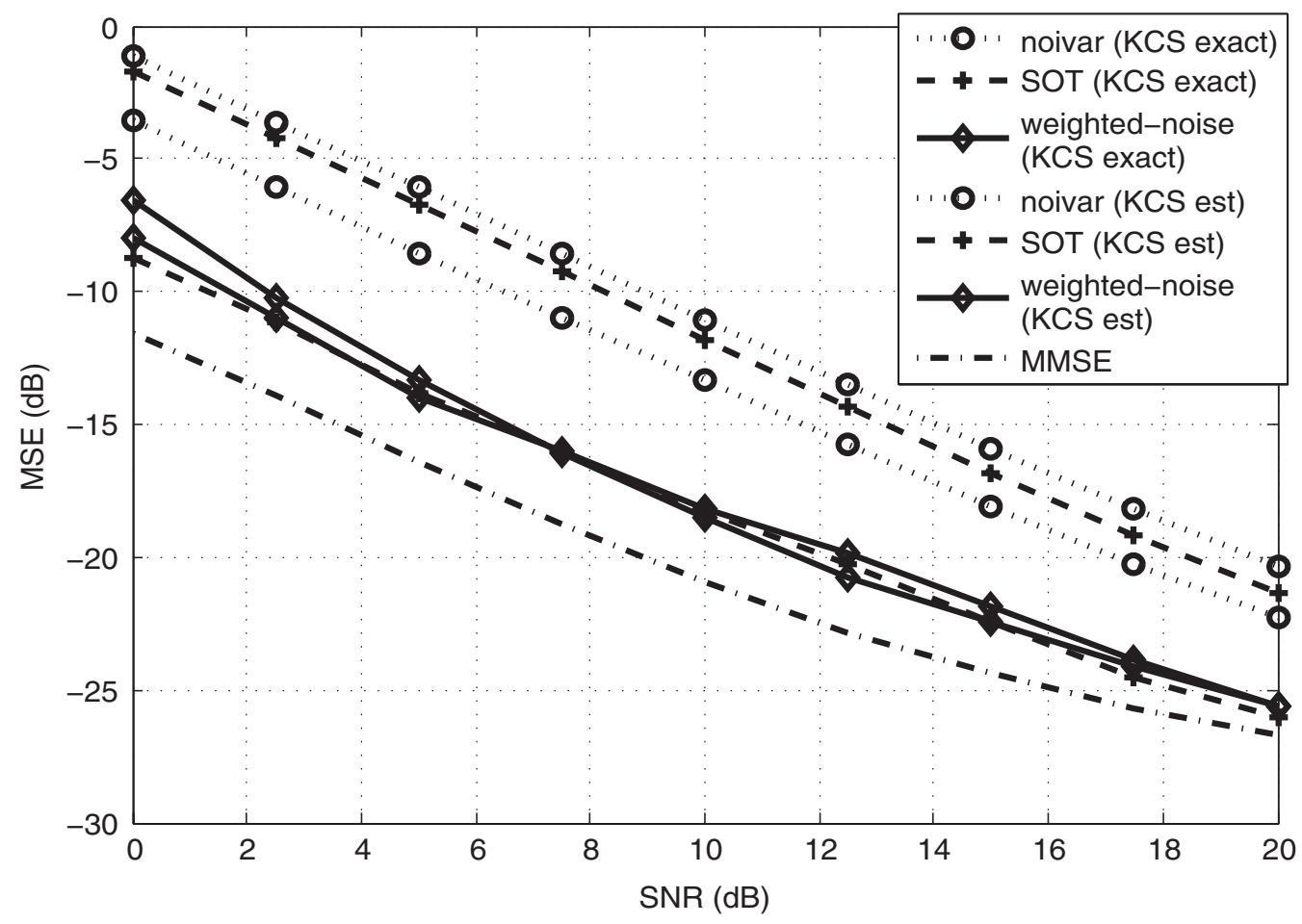

Figure 1. MSE vs. SNR plots in ITU-TU6 with exact and estimated KCS. 


$$
E\left\{\left|e_{i, n}\right|^{2} / I I\right\}=\gamma_{n}^{2}-\frac{\vartheta}{e^{\vartheta / \gamma_{n}^{2}}-1} .
$$

Substituting (11) to (14) in (10), the MSE expression is further simplified in Rosati et al (2012), which finally becomes

$$
\begin{aligned}
& M S E=\sum_{n \in C}\left[e^{-\vartheta /\left(\gamma_{n}^{2}+\sigma_{v}^{2}\right)} \sigma_{v}^{2}+\right. \\
& \left.\left(1-e^{-\vartheta /\left(\gamma_{n}^{2}+\sigma_{v}^{2}\right)}\right)\left(\gamma_{n}^{2}-\frac{\vartheta}{e^{\vartheta / \gamma_{n}^{2}}-1}\right)\right] \\
& +\sum_{n \notin C}\left[e^{-\vartheta / \sigma_{v}^{2}}\left(\sigma_{v}^{2}+\vartheta\right)\right] .
\end{aligned}
$$

The threshold in Rosati et al (2012) is derived by minimizing the MSE expression in (15) and using the assumption of $\sigma_{v}^{2}<<\gamma_{n}^{2}$. Further, assuming a uniform channel-tap power profile, $\gamma_{n}^{2}=\frac{1}{N_{t}}, n \in C$, where $N_{t}$ is the number of significant channel taps, the simplified thresholds of Oliver et al (2008) and Rosati et al (2012) are given in table 1. Observing these expressions, it is understood that they require estimation of $N_{t}, L, \sigma_{v}^{2}$. If their actual values are available, they render optimal MSE performance. However, if these parameters are estimated, the MSE performance degrades severely from that of the MMSE. This can be observed from figure 1, discussed in section 5. This inevitable MSE degradation, which implies suboptimal behavior, is the drawback of existing optimal thresholds.

\section{Modified HTP: weighted-noise threshold}

To overcome the MSE degradation, we propose a weighted-noise threshold that incorporates a flexible noise-weight parameter. To derive this threshold, the HTP interpretation of $\hat{h}_{i, n}$ in (8) is modified using a weighted-noise term defined as $\bar{v}_{i, n}=\sqrt{q} v_{i, n}$, where $q$ is the noise-weight parameter. $q$ is the scaling factor of the noise variance of each tap $n$. The modified HTP is framed as

$$
\begin{aligned}
& \left|\hat{h}_{i, n}\right|^{2}= \\
& \left\{\begin{array}{l}
\left|\bar{v}_{i, n}+v_{i, n}\right|^{2} \sim \mathrm{E}\left(\frac{1}{(q+1) \sigma_{v}^{2}}\right) \quad\left|h_{i, n}\right|^{2} \leq\left|\bar{v}_{i, n}\right|^{2}: H_{0} \\
\left|h_{i, n}+v_{i, n}\right|^{2} \sim \mathrm{E}\left(\frac{1}{\gamma_{n}^{2}+\sigma_{v}^{2}}\right) \quad\left|h_{i, n}\right|^{2}>\left|\bar{v}_{i, n}\right|^{2}: H_{1} .
\end{array}\right.
\end{aligned}
$$

The statistics of $\bar{v}_{i, n}$ are $\bar{v}_{i, n} \sim N_{c}\left(0, q \sigma_{v}^{2}\right)$. From (16), observe that on hypothesis $H_{0}$, minimum value of $h_{i, n}$ is restricted to $\sqrt{q} v_{i, n}$ unlike zero in the case of conventional HTP interpretation shown in (8). Modified HTP compares channel-tap power with $q$ times the noise power unlike comparison with zero in original HTP. Note that if $q=0$, (16) reduces to (8). With modified HTP, $P_{D}$ remains the same as (11), but $P_{F A}$ is obtained as

$$
P_{F A}=\operatorname{Pr}\left\{\left|\hat{h}_{i, n}\right|^{2}>\vartheta /\left|h_{i, n}\right|^{2}<\left|v_{i, n}\right|^{2}\right\}=\left(e^{-\frac{\vartheta}{(q+1) \sigma_{v}^{2}}}\right)
$$


To derive the weighted-noise threshold, consider Neyman-Pearson likelihood ratio test (LRT) condition given as

$$
\frac{f\left(\left|\hat{h}_{i, n}\right|^{2} / H_{1}\right)}{f\left(\left|\hat{h}_{i, n}\right|^{2} / H_{0}\right)}=\Lambda>\eta, \quad \int_{\eta}^{\infty} f\left(\Lambda / H_{0}\right) d \Lambda=P_{F A},
$$

where $f\left(\left|\hat{h}_{i, n}\right|^{2} / H_{0}\right)$ and $f\left(\left|\hat{h}_{i, n}\right|^{2} / H_{1}\right)$ are the conditional pdfs of $\left|\hat{h}_{i, n}\right|^{2}$ on $H_{0}$ and $H_{1}$ respectively. Substituting them in (18), we obtain

$$
\frac{\frac{1}{\gamma_{n}^{2}+\sigma_{v}^{2}} e^{-\left|h_{i, n}\right|^{2} /\left(\gamma_{n}^{2}+\sigma_{v}^{2}\right)}}{\frac{1}{(q+1) \sigma_{v}^{2}} e^{-\left|h_{i, n}\right|^{2} /\left((q+1) \sigma_{v}^{2}\right)}}>\eta \text {. }
$$

Rearranging the terms, (19) simplifies to (20).

$$
\left|h_{i, n}\right|^{2}>\frac{(q+1) \sigma_{v}^{2}\left(\gamma_{n}^{2}+\sigma_{v}^{2}\right)}{\gamma_{n}^{2}-q \sigma_{v}^{2}} \ln \left(\frac{\eta\left(\gamma_{n}^{2}+\sigma_{v}^{2}\right)}{(q+1) \sigma_{v}^{2}}\right)=\vartheta .
$$

The tap-detection performance improves as overlap between pdfs on both hypotheses reduces (Kay 1993). Hence it is assumed that $(q+1) \sigma_{v}^{2}<<\gamma_{n}^{2}+\sigma_{v}^{2}$. The number of pilots in any OFDM symbol is large enough to assume $\sigma_{v}^{2}<<\gamma_{n}^{2}$, even for low SNR values (Rosati et al 2012). Hence the term $\left(\gamma_{n}^{2}+\sigma_{v}^{2}\right)$ in (20) can be approximated as $\gamma_{n}^{2}$. However, for the assumption $(q+1) \sigma_{v}^{2}<<\gamma_{n}^{2}+\sigma_{v}^{2}$ to be satisfied, $q$ must satisfy the constraint $q<<\frac{\gamma_{n}^{2}}{\sigma_{v}^{2}}$. With the above said approximation, the terms $\left(\gamma_{n}^{2}+\sigma_{v}^{2}\right)$ and $\left(\gamma_{n}^{2}-q \sigma_{v}^{2}\right)$ in (20) can be canceled. Choosing $\eta=1+\frac{(q+1) \sigma_{v}^{2}}{\sigma_{v}^{2}+\gamma_{n}^{2}}$ in (20), the $\ln ($.$) term becomes strictly positive. The resulting threshold \vartheta$ is given in (21), which thereby constrains $P_{F A}$ of (17) to $P_{F A}=\frac{(q+1) \sigma_{v}^{2}}{(q+2) \sigma_{v}^{2}+\gamma_{n}^{2}}$.

$$
\left|h_{i, n}\right|^{2}>(q+1) \sigma_{v}^{2} \ln \left(\frac{\gamma_{n}^{2}+(q+2) \sigma_{v}^{2}}{(q+1) \sigma_{v}^{2}}\right)=\vartheta .
$$

Assuming uniform channel-tap power like in Rosati et al (2012), the weighted-noise threshold, (21) becomes

$$
\vartheta=(q+1) \sigma_{v}^{2} \ln \left(\frac{(q+2)}{(q+1)}+\frac{1}{(q+1) \sigma_{v}^{2} N_{t}}\right) .
$$

Note that when $q=0$, this threshold reduces to that of Oliver et al (2008) obtained with unmodified HTP. Next, we consider the analytical MSE expression of the CE incorporating weighted-noise threshold. The general expression for MSE is given as

$$
\begin{gathered}
M S E=\sum_{n \in C}\left(P_{D} E\left\{\left|e_{i, n}\right|^{2} / I\right\}\right. \\
\left.+\left(1-P_{D}\right) E\left\{\left|e_{i, n}\right|^{2} / I I\right\}\right) \\
+\sum_{n \in C}\left(P_{F A} E\left\{\left|e_{i, n}\right|^{2} / I I I\right\}\right. \\
\left.+\left(1-P_{F A}\right) E\left\{\left|e_{i, n}\right|^{2} / I V\right\}\right) .
\end{gathered}
$$


The MSE in (23) shows that all four events contribute to MSE unlike only first three contributing to MSE in (10). The first two conditional-expectations in both these MSE expressions are the same as those in (13) and (14). However, the conditional-expectation on event III is different due to the definition of $\bar{v}_{i, n}$ on modified $H_{0}$ in (16). Event IV did not cause any error on original HTP, whereas on modified HTP, it does. The conditional-expectations on events III, IV are given in (24) and (25) respectively, which are derived in Appendix section of this paper.

$$
\begin{gathered}
E\left\{\left|v_{i, n}\right|^{2} / I I I\right\}=(q+1) \sigma_{v}^{2}+\vartheta \\
E\left\{\left|e_{i, n}\right|^{2} / I V\right\}=\left(q \sigma_{v}^{2}-\frac{\vartheta}{e^{\vartheta /\left(q \sigma_{v}^{2}\right)}-1}\right) .
\end{gathered}
$$

Substituting all the conditional-expectations in (23), the simplified MSE expression of the weighted-noise threshold is given in (26). Note that if $q=0$, MSE in (26) reduces to (15) of original HTP.

$$
\begin{aligned}
& M S E=\sum_{n \in C}\left[e^{-\vartheta /\left(\gamma_{n}^{2}+\sigma_{v}^{2}\right)} \sigma_{v}^{2}+\left(1-e^{-\vartheta /\left(\gamma_{n}^{2}+\sigma_{v}^{2}\right)}\right)\left(\gamma_{n}^{2}-\frac{\vartheta}{e^{\vartheta / \gamma_{n}^{2}}-1}\right)\right] \\
& \quad+\sum_{n \notin C}\left[e^{-\vartheta /\left((q+1) \sigma_{v}^{2}\right)}\left((q+1) \sigma_{v}^{2}+\vartheta\right)+\right. \\
& \left.\quad\left(1-e^{-\vartheta /\left((q+1) \sigma_{v}^{2}\right)}\right)\left(q \sigma_{v}^{2}-\frac{\vartheta}{e^{\vartheta /\left(q \sigma_{v}^{2}\right)}-1}\right)\right] .
\end{aligned}
$$

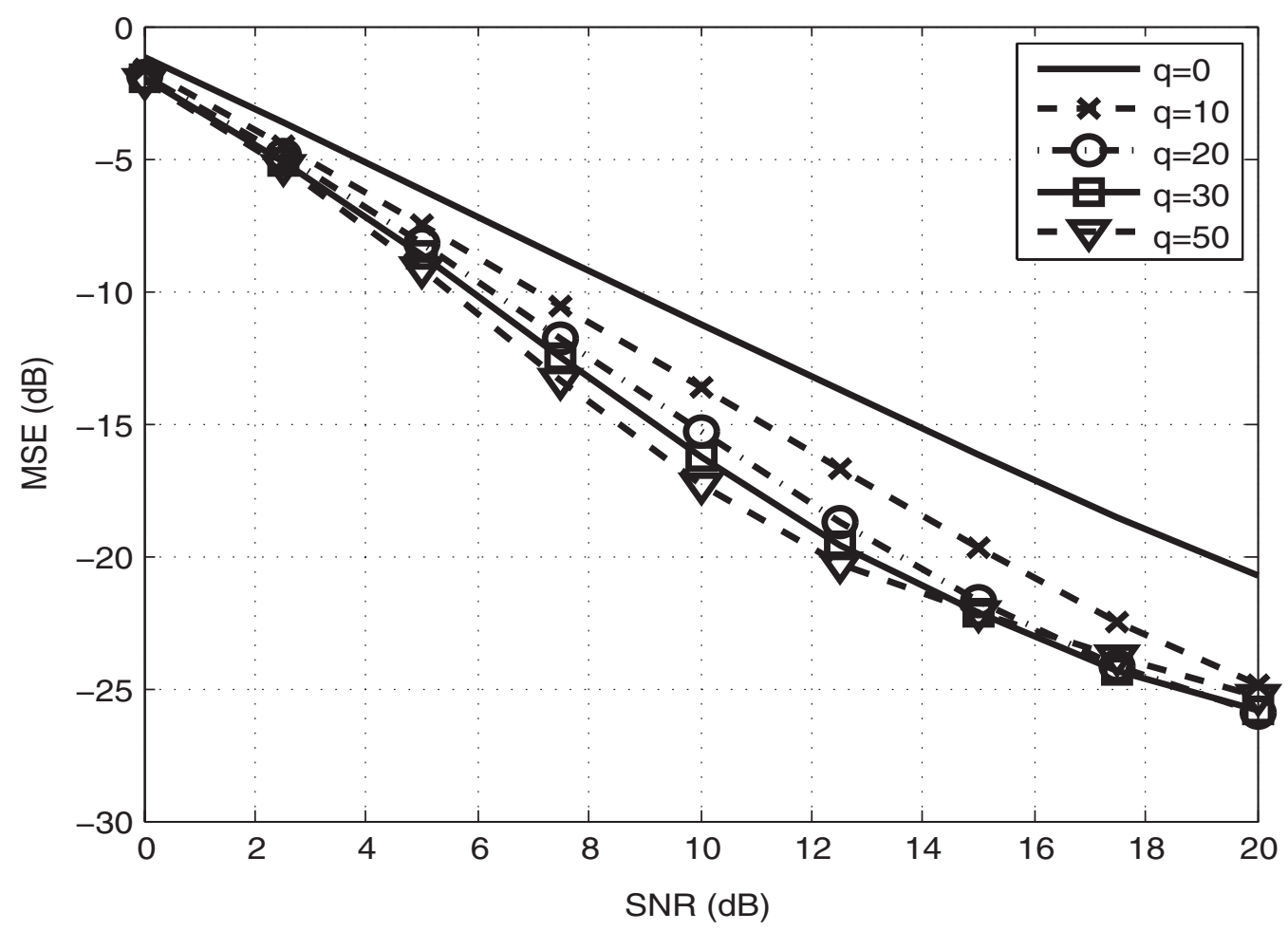

Figure 2. MSE vs. SNR plots in ITU-TU6 with exact and estimated KCS. 
Comparing the proposed weighted-noise threshold (22), with those in table 1, it can be observed that (22) involves a flexible parameter $q$ in addition to the KCS parameters required in all threshold expressions. Parameter $q$ is flexible because, choice of $q$ value can be controlled to achieve better MSE performance, more close to that of MMSE compared to the existing thresholds as discussed later.

\section{Numerical and system simulation results}

We consider an OFDM system with $N=1024$ subcarriers carrying QPSK modulated data symbols. The pilots are arranged in comb-type fashion with a ratio of $N / 8$. The cyclic prefix has a length of $N / 4$ symbols. Rayleigh fading ITU-TU6 (Rosati et al 2012) and WiMAX-SUI4 channels with a maximum Doppler frequency of $20 \mathrm{~Hz}$, which is invariant within one OFDM-symbol is considered. These correlated rayleigh fading channels are simulated using the methodology described in Zhinian \& Wenjun (2007).

\subsection{Study of KCS estimation on MSE}

First, we study the impact of KCS parameter estimation on MSE performance of the CE in ITUTU6 channel environment. Noise variance $\sigma_{v}^{2}$ is estimated as suggested in Kang et al (2007). Channel length $(L=100)$ is estimated as the delay, at which autocorrelation of LS-estimated CIR $\hat{h}_{i, n}^{L S}$ decays considerably. Actual value of $N_{t}=6$, but $N_{t}=L / 3$ is considered, to see

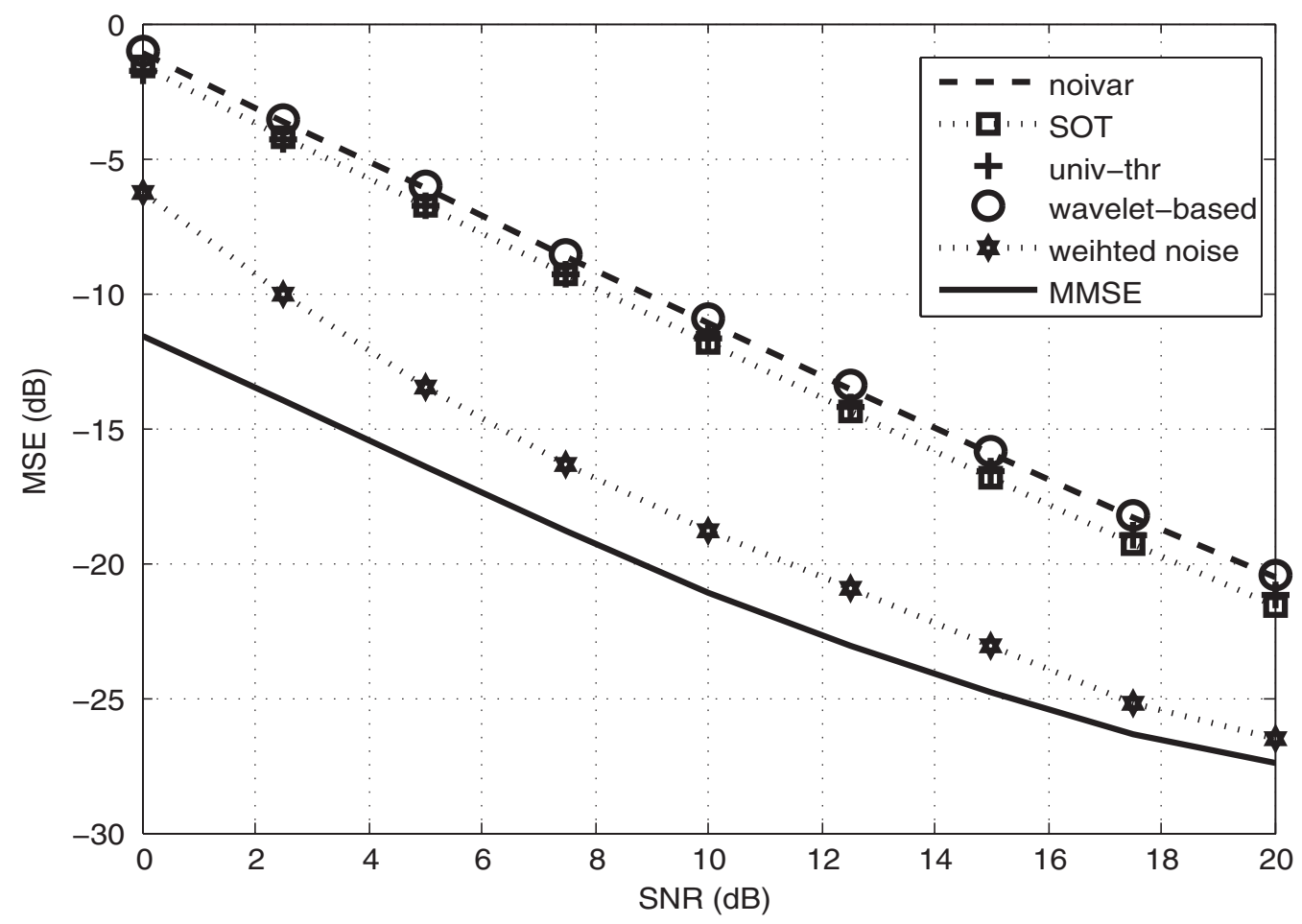

Figure 3. MSE vs. SNR plot in ITU-TU6 rayleigh fading channel with a doppler of $20 \mathrm{~Hz}$. 
the effect of worst approximation. Using exact and estimated KCS, the numerical results of analytical MSE performances are obtained. For thresholds in Kang et al (2007) and Rosati et al (2012), analytical MSE in (15) and for the proposed threshold, analytical MSE in (26) are used. The plots are shown in figure 1, with the following labels.

1. noivar - Threshold given in Kang et al (2007)

2. SOT - Suboptimal threshold derived in Rosati et al (2012)

3. weighted-noise - Proposed threshold derived as (22)

4. MMSE - The benchmark performance (27)

$$
M M S E=\sum_{n \in C}\left(\frac{\gamma_{n}^{2}}{1+\frac{\gamma_{n}^{2}}{\sigma_{v}^{2}}}\right) .
$$

The benchmark MMSE expression is given in (27). This is derived in Rosati et al (2012), by assuming that the significant channel taps are perfectly separated from the noise taps. In the simulations, the MMSE plot is obtained by informing the CE, the exact positions of significant channel taps. The results in figure 1 show that, incorporating the thresholds in Kang et al (2007) and Rosati et al (2012), if KCS are estimated, the MSE performance degrades significantly from that when exact KCS is known. However, for the proposed threshold, if KCS are estimated or exactly known, MSE performance degradation is negligible. Also the MSE performance with the proposed threshold is much better than that obtained with others compared, whether KCS

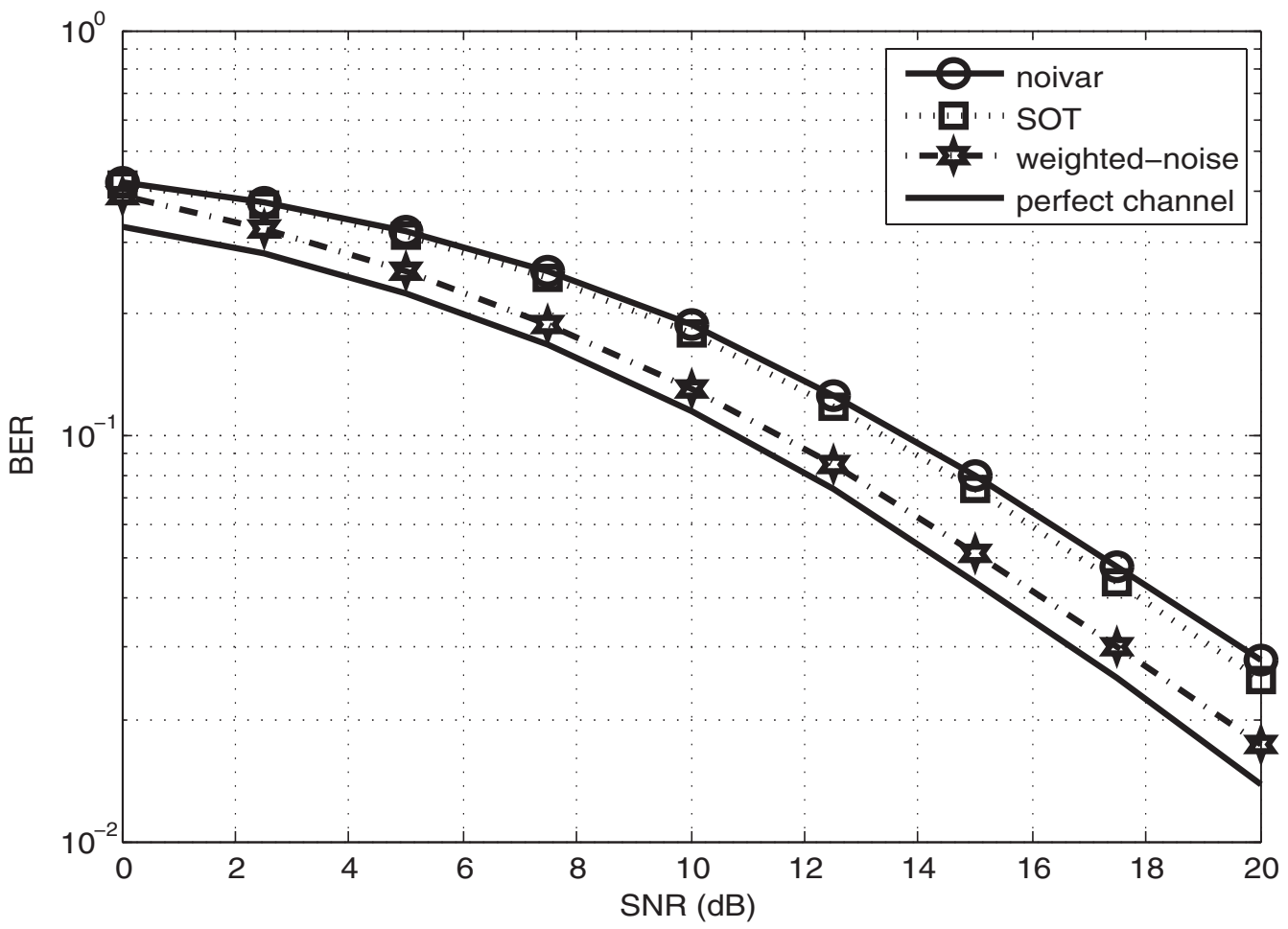

Figure 4. BER vs. SNR plot in ITU-TU6 rayleigh fading channel with a doppler of $20 \mathrm{~Hz}$. 
is estimated or exactly known. Note that the value of $q=30$ is used in the proposed threshold expression.

Estimating $\sigma_{v}^{2}, N_{t}, L$ as before, in a ITU-TU6 channel environment, MSE plots are obtained with the proposed threshold, by varying the parameter $q$. These plots are given in figure 2 , which show that for $q=30$, better MSE vs. SNR performance is obtained. The MSE performance is controlled with flexible parameter $q$. This controllability is not present in any of the existing thresholds developed so far in the literature. Proper choice of $q$ renders better MSE performance even with estimation of all the KCS parameters as shown already in figure 1 . Notice that the MSE performance obtained with $q=30$ is much better than that with $q=0$, which represents the optimal threshold derived in Oliver et al (2008).

\subsection{MSE and bit error rate (BER) performance comparisons}

Consider the OFDM system simulated in ITU-TU6 channel environment. The simulated MSE performance of the CE using estimated KCS parameters (discussed in section 5.1) is shown in figure 3, with additional labels:

1. wavelet-based - Threshold derived in Lee et al (2009)

2. univ-thr - Threshold given in Xie et al (2013)

Figure 3 confirms that proposed threshold renders better MSE performance to the CE than existing optimal ones. The OFDM system BER performance simulations are shown in figure 4 . The

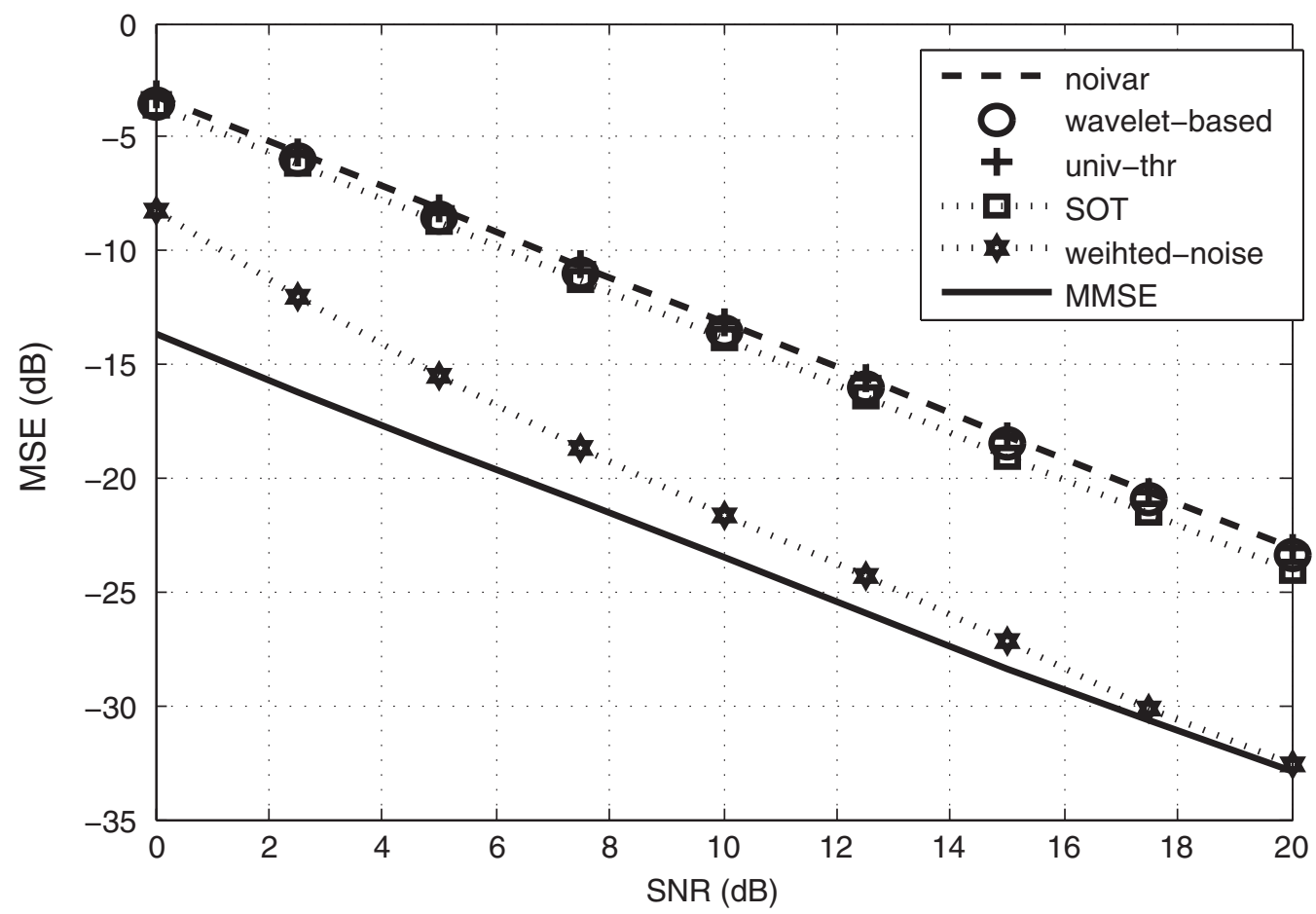

Figure 5. MSE vs. SNR plot in WiMAX SUI4 rayleigh fading channel with a doppler of $20 \mathrm{~Hz}$. 
benchmark for BER plots is the BER obtained with the CE having complete channel knowledge (label: perfect channel). The results confirm that the proposed threshold outperforms all the others compared. Next, we consider the case of WiMAX SUI4 channel environment. The simulated MSE performance of CE is shown in figure 5. The system BER performance is shown in figure 6. The KCS parameters of all the incorporated thresholds are estimated as mentioned in section 5.1. These results also confirm that the proposed threshold outperforms all the existing thresholds compared. From the numerical results in section 5.1, and system simulations shown in this section, it is understood that the proposed threshold renders better MSE performance to the $\mathrm{CE}$ than the others compared. However, this is possible only by substituting optimal value for $q$, whose computation is discussed next.

\subsection{Empirical formula for $q$}

The proposed threshold renders better MSE and BER performances with the help of optimal $q$ value. We attempt to give an empirical formula for $q$ as the ratio of maximum channel-tap power to average channel power in one OFDM symbol duration. For example, in a three tap channel with gains, 1, 0.398, 0.158, with $L=33$, average channel power is $\frac{\left(1+0.398^{2}+0.158^{2}\right)}{33}=0.0358$, $q=\frac{1}{0.0398}=27.88$. From this discussion, one may be under the impression that computation of $q$ value requires the actual channel power profile. However, it is not required. In the channel estimation procedure steps shown in section 2, in step 4, we obtain the LS estimated and

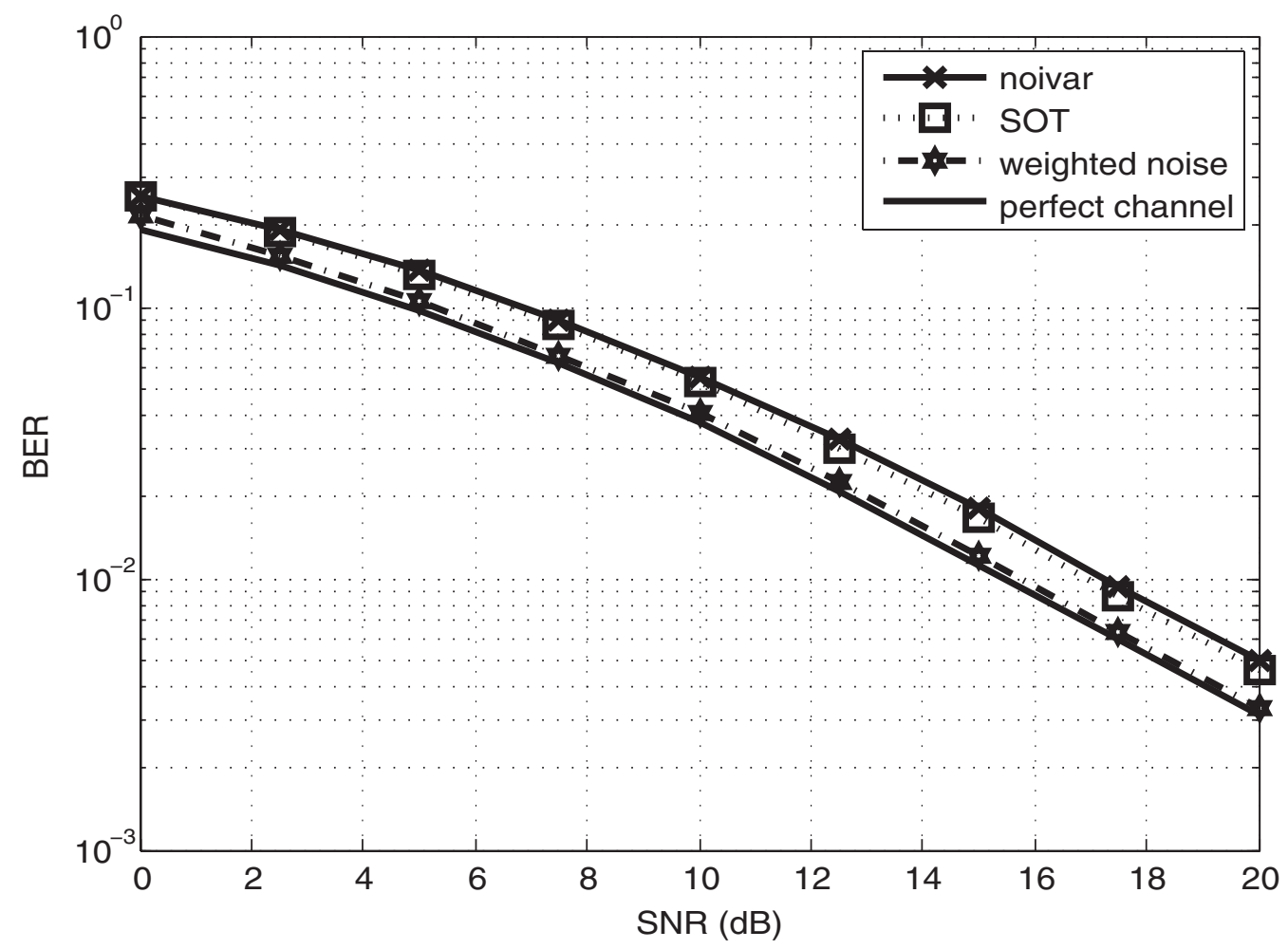

Figure 6. BER vs. SNR plot in WiMAX SUI4 rayleigh fading channel with a doppler of $20 \mathrm{~Hz}$. 
truncated CIR, $\hat{h}_{i, n}$. Using $\hat{h}_{i, n}, 0 \leq n \leq L-1$ as the channel power profile, an initial value of $q$ can be estimated using the empirical formula. The value of $q$ can be thereafter tuned for better MSE performance if necessary.

For four different channels, empirical $q$ value obtained from the actual channel tap power profile, best $q$ value obtained from simulations, and the initial estimate of $q$ at an SNR of $0 \mathrm{~dB}$ (computed using $\hat{h}_{i, n}, 0 \leq n \leq L-1$ as the channel power profile) are shown in table 2 . The table shows that the initial estimate of $q$ is almost same as the best $q$ obtained from the simulations. Also the empirical formula for $q$ provides a good approximation to the best value of $q$ obtained from the simulations. In some channel environments (e.g., ITU-TU6), initial estimate of $q$ matches to the best $q$ obtained from simulations. Hence, further tuning of $q$ is not necessary because the initial estimate of $q$ itself is the best $q$. However in all channel environments, this may not be possible and a systematic tuning procedure for $q$ may become necessary.

Table 2. Empirical $q$, simulated $q$, estimated $q$ and factor.

\begin{tabular}{lcccc}
\hline Channel & Empirical $q$ & Simulated $q$ & Initial estimate of $q$ & Factor $=\frac{\left(\gamma_{n}^{2}+\sigma_{v}^{2}\right)}{\left(\gamma_{n}^{2}-q \sigma_{v}^{2}\right)}$ \\
\hline ITU-TU6 & 35 & 30 & 29.8 & 1.1 \\
WiMax SUI-4 & 27.88 & 20 & 26.5 & 1.058 \\
Brazil E & 13.66 & 20 & 18.4 & 1.091 \\
Brazil A & 119.3 & 100 & 105 & 1.313 \\
\hline
\end{tabular}

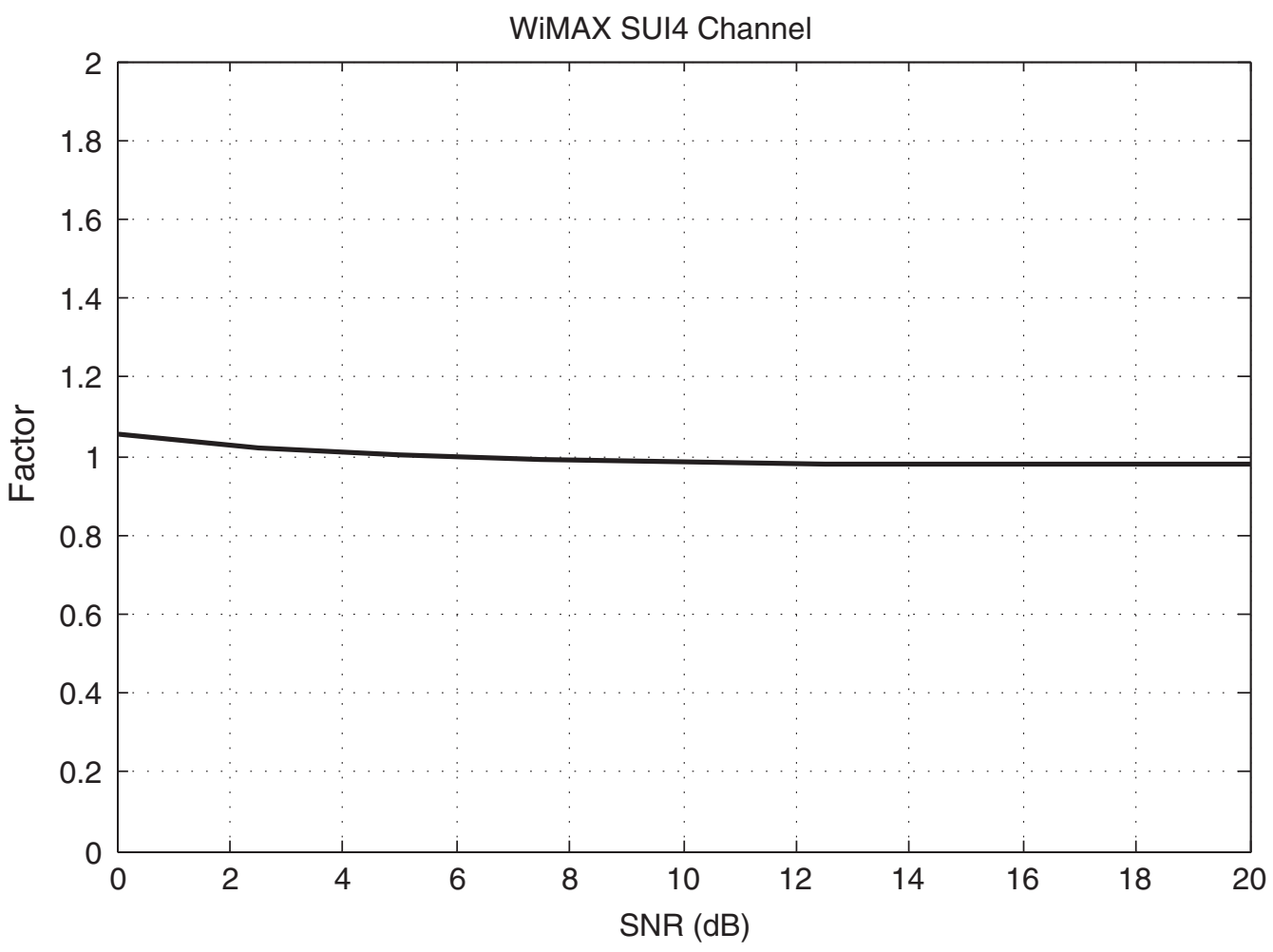

Figure 7. Factor vs. SNR plot in WiMAX SUI4 rayleigh fading channel with a doppler of $20 \mathrm{~Hz}$. 
Development of such a tuning procedure is possible, for example using a suitable information theoretic criteria, which forms the future scope of this paper.

\subsection{Constraint on $q$}

Here, it is justified that the empirical $q$ value computed as discussed in 5.3, always ensures $q<<$ $\frac{\gamma_{n}^{2}}{\sigma_{v}^{2}}$, which is essential to cancel the terms $\left(\gamma_{n}^{2}+\sigma_{v}^{2}\right)$ and $\left(\gamma_{n}^{2}-q \sigma_{v}^{2}\right)$ in (20). The justification is as follows:

The empirical $q$ for a channel with uniform power profile, namely a channel with $\gamma_{n}^{2}=\frac{1}{N_{t}}$ is given in (28). From Rosati et al (2012), the fraction $\frac{\gamma_{n}^{2}}{\sigma_{v}^{2}}$ can be expressed as $\frac{\rho N_{p}}{N_{t}}$, where $N_{p}$ is large enough to ensure the inequality $\frac{1}{L}<<\frac{\rho N_{p}}{N_{t}}$. This justifies that the value of the fraction $\frac{\left(\gamma_{n}^{2}+\sigma_{v}^{2}\right)}{\left(\gamma_{n}^{2}-q \sigma_{v}^{2}\right)}$ in (20) is approximately 1 , for any given SNR value. However, for completeness, the verification of this fraction being 1 is also verified from the system simulations.

$$
q=\frac{N_{t}\left(\frac{1}{N_{t}}+\frac{1}{N_{t}}+\ldots N_{t} \text { times }\right)}{L}=\frac{1}{L}
$$

In WiMAX SUI4 and ITU-TU6 channel environments, the plot of $\frac{\left(\gamma_{n}^{2}+\sigma_{v}^{2}\right)}{\left(\gamma_{n}^{2}-q \sigma_{v}^{2}\right)}$ (called as Factor in the plots) vs. SNR is shown in figure 7 and 8 respectively. It can be seen from the figures

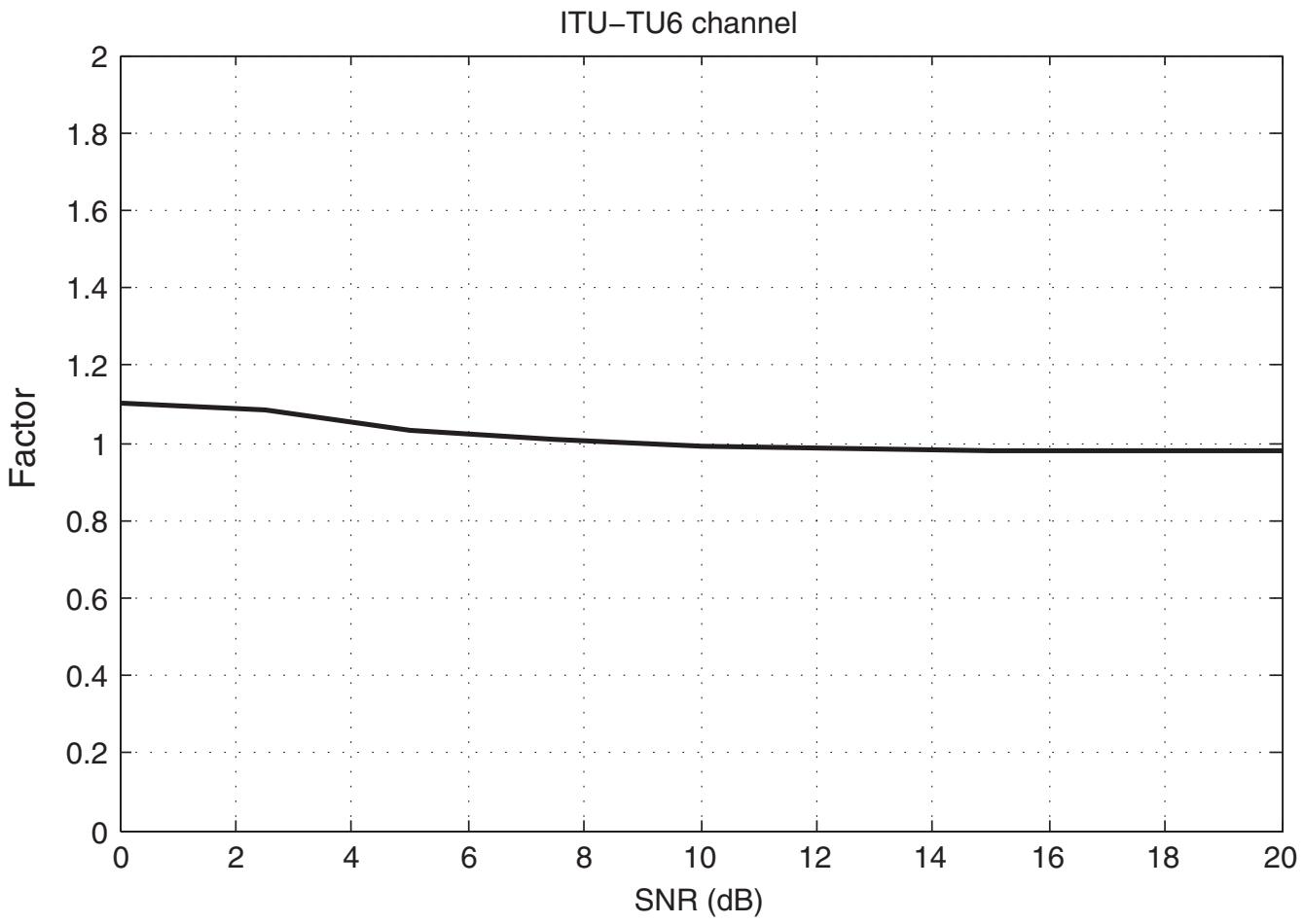

Figure 8. Factor vs. SNR plot in ITU-TU6 rayleigh fading channel with a doppler of $20 \mathrm{~Hz}$. 


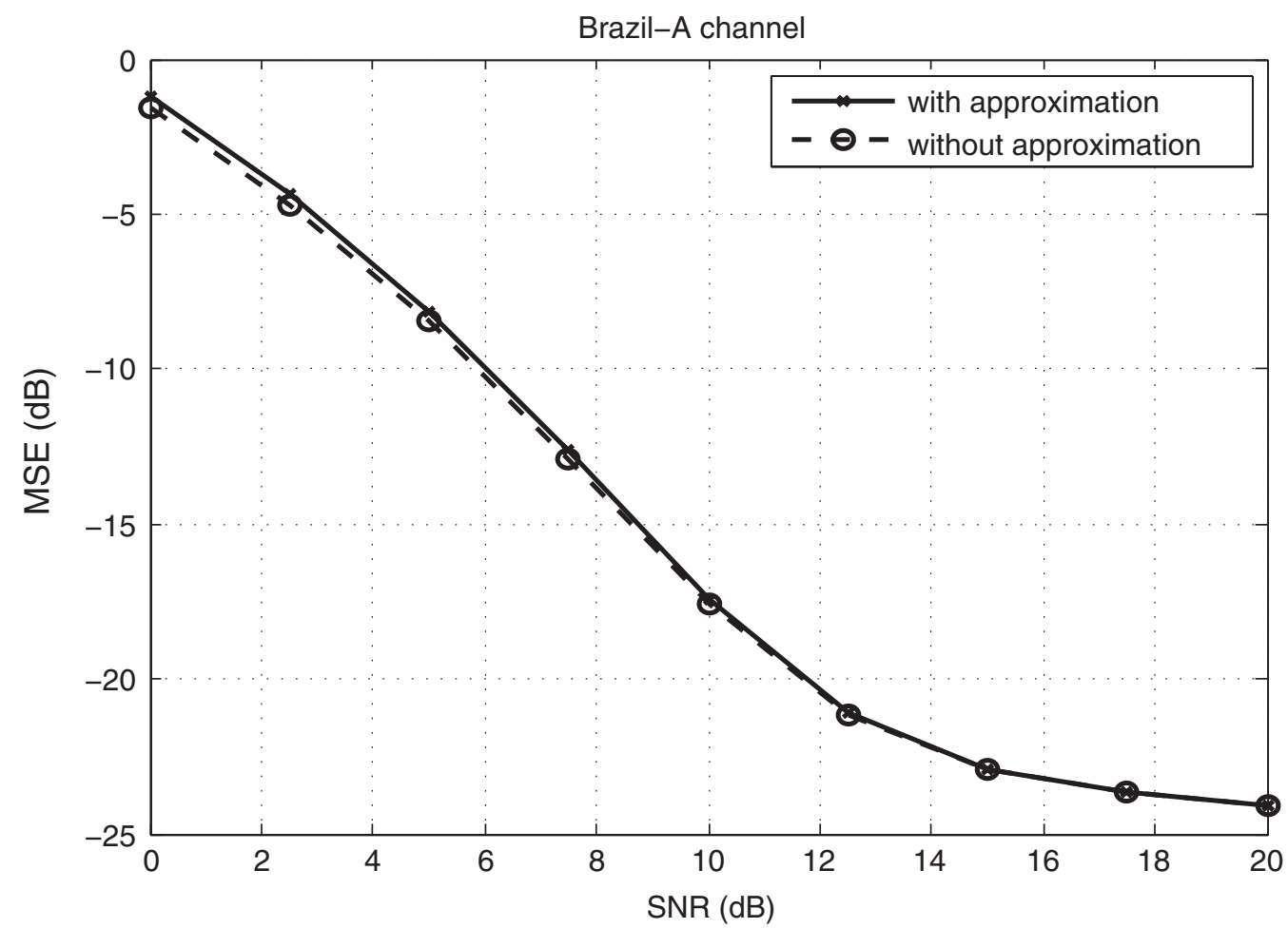

Figure 9. MSE vs. SNR plot in Brazil-A rayleigh fading channel with a doppler of $20 \mathrm{~Hz}$.

that the Factor is approximately 1, for all SNR values. Further, the value of this Factor at SNR of $0 \mathrm{~dB}$ is shown for various channels in table 2. The Table shows that for Brazil-A channel the Factor value appears to deviate from 1, by a significant amount. Hence, for this channel, without making the approximation of Factor as 1 in (20) and by making the Factor as 1, the simulations of MSE vs. SNR are shown in figure 9. The plots show that the MSE deviation is negligible. Thus the threshold expression given in (22) is a good approximation and is suitable for all practical OFDM systems.

\section{Conclusion}

In this paper, LS based channel estimation using denoising thresholds is considered for OFDM based wireless systems like DVB, LTE, WiMAX, WiFi, WLAN and B3G. Existing time-domain denoising thresholds involve the estimation of one or more KCS parameters, introducing degradation in MSE performance of the CE. To combat this degradation, a weighted-noise threshold is proposed and developed. For this purpose, a modified HTP interpretation of the LS estimated CIR is proposed. Analytical MSE expression of the CE with the proposed threshold is also derived. OFDM system simulations are carried out in rayleigh fading ITU-TU6 and WiMAXSUI4 channel environments. The MSE performance results of the CE and BER performance results of OFDM system revealed that the proposed threshold indeed outperforms the existing state-of-art approaches. 


\section{Appendix: proof of conditional expectations (24) and (25)}

With the modified hypothesis shown in (16), the modified event III, namely $\left(\left|\hat{h}_{i, n}\right|^{2}>\vartheta\right) /\left(\left|h_{i, n}\right|^{2} \leq\left|v_{i, n}\right|^{2}\right)$ occurs with a probability of $P_{F A}$. In this case, though actual tap is not present, the threshold detects as tap is existing, thus causing a squared error of $\left|\bar{v}_{i, n}+v_{i, n}\right|^{2}$. The mean of this squared error is given in (29) and (30).

$$
\begin{gathered}
E\left\{\left|e_{i, n}\right|^{2} / I I I\right\}=E\left\{\left|\bar{v}_{i, n}+v_{i, n}\right|^{2} / I I I\right\}=E\{x / I I I\} \\
E\{x / I I I\}=\int_{-\infty}^{\infty} x f(x / I I I) d x .
\end{gathered}
$$

Using Baye's theorem, the conditional density function is given in (31).

$$
f(x / I I I)=\frac{f(x, I I I)}{f(I I I)=\int_{-\infty}^{\infty} f(x, I I I) d x} .
$$

Simplifying (31), we get (32), which is used in (30), to finally obtain (24).

$$
\begin{aligned}
& f(x / I I I)=\frac{\frac{1}{(q+1) \sigma_{v}^{2}} e^{-x /\left((q+1) \sigma_{v}^{2}\right)}}{\int_{\vartheta}^{\infty} \frac{1}{(q+1) \sigma_{v}^{2}} e^{-x /\left((q+1) \sigma_{v}^{2}\right) d x}}, \vartheta \leq x<\infty \\
& =\frac{\frac{1}{(q+1) \sigma_{v}^{2}} e^{-x /\left((q+1) \sigma_{v}^{2}\right)}}{e^{-\vartheta /\left((q+1) \sigma_{v}^{2}\right)}} .
\end{aligned}
$$

On similar lines as above, modified event $I V,\left(\left|\hat{h}_{i, n}\right|^{2}<\vartheta\right) /\left(\left|h_{i, n}\right|^{2} \leq\left|v_{i, n}\right|^{2}\right)$ has a probability of $\left(1-P_{F A}\right)$. In this case, the actual tap is not present, and the threshold also detects as no tap. Though the threshold identified correct, due to modified $H_{0}$ in (16), a squared error of $\left|\bar{v}_{i, n}\right|^{2}$ exists. The mean of this squared error is simplified similar to that on event III to finally obtain (25). The conditional density function used in deriving (25) is (33).

$$
\begin{aligned}
& f(x / I V)=\frac{\frac{1}{q \sigma_{v}^{2}} e^{-x /\left(q \sigma_{v}^{2}\right)}}{\int_{0}^{\vartheta} \frac{1}{q \sigma_{v}^{2}} e^{-x /\left(q \sigma_{v}^{2}\right)} d x}, 0 \leq x \leq \vartheta \\
& =\frac{\frac{1}{q \sigma_{v}^{2}} e^{-x /\left(q \sigma_{v}^{2}\right)}}{1-e^{-\vartheta /\left(q \sigma_{v}^{2}\right)}} .
\end{aligned}
$$

\section{References}

Carlos Augusto, Rocha ACdS and Luciano Leonel Mendes 2007 Performance analysis of channel estimation schemes for OFDM systems. International workshop on telecommunications-IWT. pp 32-36

Coleri S, Ergen M, Puri A and Bahai A 2002 Channel estimation techniques based on pilot arrangement in OFDM systems. IEEE Trans. Broadcasting 48(3): 223-229, doi: 10.1109/TBC.2002.804034 
Dai L, Wang Z and Yang Z 2013 Spectrally efficient time-frequency training OFDM for mobile large-scale MIMO systems. IEEE J. Selected Areas Commun. 31(2): 251-263

Hsieh M H and Wei C H 1998 Channel estimation for OFDM systems based on comb-type pilot arrangement in frequency selective fading channels. IEEE Trans. Consumer Electron. 44(1): 217-225, doi: $10.1109 / 30.663750$

Kang Y, Kim K and Park H 2007 Efficient DFT-based channel estimation for OFDM systems on multipath channels. IET Commun 1(2): 197-202, doi: 10.1049/iet-com:20050337

Kashyap S and Mehta N B 2014 Optimal binary power control for underlay CR with different interference constraints and impact of channel estimation errors. IEEE Trans. Commun. 62: 3753-3764

Kay S M 1993 Fundamentals of statistical signal processing, vol. 2, Detection theory. Prentice Hall

Larsson E G, Liu G, Li J and Giannakis G B 2001 Joint symbol timing and channel estimation for OFDM based WLANs. IEEE Comm. Lett. 5(8): 325-327

Lee Y S, Shin H C and Kim H N 2009 Channel estimation based on a time-domain threshold for OFDM systems. IEEE Trans. Broadcasting 55(3): 656-662, doi: 10.1109/TBC.2009.2027575

Minn H and Bhargava V 2000 An investigation into time-domain approach for OFDM channel estimation. IEEE Trans. Broadcasting 46(4): 240-248, doi: 10.1109/11.898744

Morelli M and Mengali U 2001 A comparison of pilot-aided channel estimation methods for OFDM systems. IEEE Trans. Signal Process. 49(2): 3065-3073

Oliver J, Aravind R and Prabhu K M M 2008 Sparse channel estimation in OFDM systems by thresholdbased pruning. IEEE Electron. Lett. 44(13): 830-832, doi: 10.1049/el:20081089

Rosati S, Corazza G and Vanelli-Coralli A 2012 OFDM channel estimation based on impulse response decimation: Analysis and novel algorithms. IEEE Trans. Commun. 60(7): 1996-2008, doi: 10.1109/TCOMM.2012.051012.090606

Sheng B 2014 Blind timing synchronization in OFDM systems by exploiting cyclic structure. Trans. Emerging Telecommun. Technol. 25(2): 155-160, doi: 10.1002/ett.2563

van de Beek J J, Edfors O, Sandell M, Wilson S and Ola Borjesson P 1995 On channel estimation in OFDM systems. In: IEEE 45th vehicular technology conference, vol 2, pp 815-819, doi: 10.1109/VETEC.1995.504981

Xie H, Andrieux G, Wang Y, Diouris J F and Feng S 2013 Efficient time domain threshold for sparse channel estimation in OFDM system. AEU-Int. J. Electron. Commun. 68: 277-281

Zhinian L and Wenjun Z 2007 Simulation for correlated rayleigh fading channels by FIR pulse-shaping filtering. In: International conference on wireless communications, Networking and Mobile Computing, pp 1091-1094, doi: 10.1109/WICOM.2007.279 